\title{
NIR-II Fluorescent Biodegradable Nanoprobe for Precise Acute Kidney/Liver Injury Imaging and Therapy
}

\author{
Xiaoguang Ge, Lichao Su, Lijiao Yang, Qinrui Fu, Qingqing Li, Xuan Zhang, \\ Naishun Liao, Huanghao Yang, and Jibin Song*
}

MOE Key Laboratory for Analytical Science of Food Safety and Biology, College of Chemistry, Fuzhou University, Fujian Science \& Technology Innovation Laboratory for Optoelectronic Information of China, Fuzhou 350108, People’s Republic of China.

Corresponding author: jibinsong@fzu.edu.cn

Table of Contents

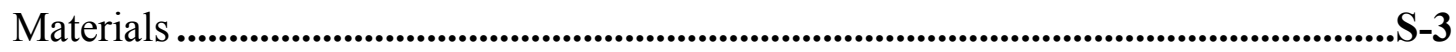

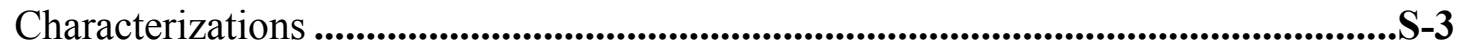

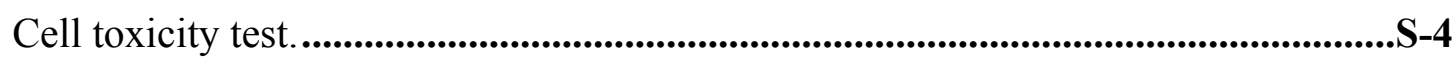

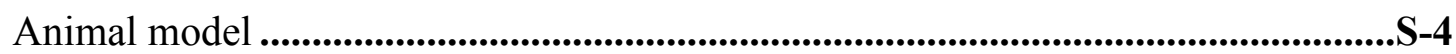

Figure S1. NIR-II fluorescence spectra of different concentration of BPQDs............S-5

Figure S2. P 2p XPS spectra of the BPQDs at day 8..........................................5-5

Figure S3. NIR-II fluorescence intensity of BPQDs solutions treated with different kinds of $\mathrm{ROS}\left(\mathrm{H}_{2} \mathrm{O}_{2}, \cdot \mathrm{OH}\right.$, and $\left.\mathrm{O}_{2}{ }^{-}\right)$...................................................................5-6

Figure S4. The scavenging efficiency of BPQDs incubated with $\mathrm{H}_{2} \mathrm{O}_{2}, \bullet \mathrm{OH}$, and $\mathrm{O}_{2}{ }^{\bullet-}$. S-6

Figure S5. The free radical scavenging efficiency of BPQDs with lapse of time......S-7

Figure S6. Cell viability of 293T and L02 incubated with BPQDs at different concentrations

Figure S7. Cell viability of $293 \mathrm{~T}$ and $\mathrm{L} 02$ under different treatment in four groups: (1) control, (2) BPQDs, (3) $\mathrm{H}_{2} \mathrm{O}_{2}$, (4) $\mathrm{H}_{2} \mathrm{O}_{2}+$ BPQDs. ..............................................

Figure S8. Confocal images of ROS scavenging in cells indicated by DCFH-DA emitting green fluorescence in L02 cells with $1 \mathrm{mM} \mathrm{H}_{2} \mathrm{O}_{2}$ S-8 Figure S9. NIR-II fluorescence images at $1050 \mathrm{~nm}$ of 96 -well plates covered by pork 
with varied thickness of $0,1,3$, and $5 \mathrm{~mm}$.

Figure S10. NIR-II fluorescence images at $1050 \mathrm{~nm}$ and $1250 \mathrm{~nm}$ of mice at post-injection $5 \mathrm{~min}$

Figure S11. In vivo NIR-II fluorescence images of mice at $6 \mathrm{~h}$ post-injection and corresponding region of interest (ROI) analysis.

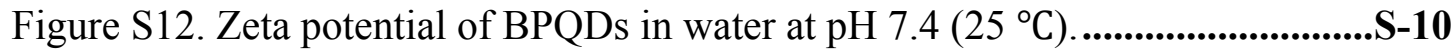

Figure S13. Ex vivo NIR-II FL images of mince treated with BPQDs (1.5 nm and10.0 $\mathrm{nm}$ ) and corresponding region of interest analysis.

Figure S14. Hematoxylin and eosin staining of main organs from mice treated with BPQDs including heart, kidney, spleen, liver.

.S-10

\section{Materials}

The black phosphorus (BP) crystalline powders (purity 99.998\%) were purchased from XFNANO Co. Ltd (Nanjing, China). N-methyl pyrrolidone (NMP), potassium persulfate, ethanol $(\mathrm{EtOH}), \quad$ sodium hydroxide $(\mathrm{NaOH})$, hydrogen peroxide $\left(\mathrm{H}_{2} \mathrm{O}_{2}\right)$, potassium persulfate, ferrous sulfate $\left(\mathrm{FeSO}_{4}\right)$, hydrochloric acid, nitric acid, glutathione, sodium hypochlorite $(\mathrm{NaClO})$ were supplied by Sinopharm Chemical Reagent Beijing Co., Ltd. riboflavin-5-phosphate sodium, acetylsalicylic acid (99\%) and aladdin2,2'-azino-bis(3-ethylbenzothiazoline-6-sulfonic acid) (ABTS) was purchased from Sigma-Aldrich. Alanine aminotransferase Assay Kit (ALT) and aspartate aminotransferase Assay Kit (AST) were obtained from NanJing Jiancheng Bioengineering Institute. The Enzyme Linked Immuno Sorbent Assay (ELISA) kits (CRE and BUN) were purchased from Shanghai Jianglai Industrial Limited By Share Ltd. Fetal bovine serum (FBS), RPMI 1640 medium, Dulbecco's eagle medium (DMEM), penicillin/streptomycin solution, Trypsin solution and phosphate buffered saline (PBS) were supplied by Gibco (Grand Island, NY). Cell Counting Kit-8 (CCK-8) were obtained from Med Chem Express (Monmouth Junction, NJ, USA). 2',7'-Dichlorofluorescin diacetate (DCFH-DA) provided by

\section{Characterizations}

Transmission electronic microscopy (TEM) images were performed on an HT7700 transmission electron microscope (HITACHI, Japan) at $100 \mathrm{kV}$. Dynamic 
light scattering (DLS) and zeta potential were tested by a Malvern Zetasizer Nano ZS (Malvern, U.K.). Ultraviolet-visible-near-infrared light (UV-Vis-NIR) absorption spectra was recorded from UH4150 spectrophotometer (HITACHI, Japan). Fluorescence spectrometer FLS980 (Edinburgh Instruments, England) under an external adjustable $808 \mathrm{~nm}$ laser were employed to record NIR II fluorescence spectra. NIR-II fluorescence imaging in vivo and in vitro was detected by an In-Vivo Master small animal NIR-II bioimaging system (Wuhan Grand-imaging Technology Co., Ltd) equipped with thermoelectric cooled InGaAs camera (Princeton Instruments). X-ray diffraction (XRD) patterns were captured by an Ultima IV 285 X-ray powder diffractometer (Rigaku Co., Japan). X-ray photoelectron spectra (XPS) was obtained by ESCALAB 250Xi X-ray photoelectron spectrometer (Thermo scientific). Inductively coupled plasma mass spectrometry (ICP-MS) analyses were carried out on an iCAP RQ system (Thermo Fisher). The fluorescence images of cells were captured by a confocal laser scanning microscope (CLSM, Nikon A1+, Japan).

\section{Cell toxicity test.}

Human hepatocyte cell line (L02) and human embryonic kidney cell line (293T) were chosen to investigate the cell toxicity. Typically, L02 and 293T were respectively cultured with RPMI 1640 medium and DMEM containing 10\% FBS and $1 \%$ penicillin/streptomycin at $37{ }^{\circ} \mathrm{C}$ in sterile cell culture flasks at a $95 \%$ humidified atmosphere with $5 \% \mathrm{CO}_{2}$. Cells were seeded in 96 -well culture plates $\left(5 \times 10^{3}\right.$ cells/well) for $12 \mathrm{~h}$. Then, cells were incubated with different concentration of BPQDs for $24 \mathrm{~h}$ at $37^{\circ} \mathrm{C}$. The concentration of BPQDs was ranged from 0 to $100 \mu \mathrm{g} \mathrm{mL} \mathrm{m}^{-1}$. In the following, $10 \mu \mathrm{L}$ CCK-8 solution was added and cultured for $30 \mathrm{~min}$. The absorption at $450 \mathrm{~nm}$ was recorded by ELISA instrument. The results were obtained by calculating average absorption intensity at $450 \mathrm{~nm}$ of each concentration in three wells. Subsequently, the viability of cells was calculated by the following formula: Viability $(\%)=($ mean of absorbance value of treatment group/mean absorbance value of control) $\times 100 \%$. 


\section{Animal model}

All procedures and animal experiments were approved by the Institutional Animal Care and Use Committee of Fujian Medical University. BALB/c nude mice (female, 5-6 weeks old, 20 g) were obtained from Shanghai SLAC Laboratory Animal Co., Ltd. The animals were cared for in accordance with the guide for the care and use of laboratory animals in China. The mice were kept under specific-pathogen-free (SPF) controlled conditions of $22 \pm 1{ }^{\circ} \mathrm{C}$ temperature, $60 \% \pm$ 10 humidity with a $12 \mathrm{~h}$ light/dark cycle and free access to standard rodent diet and water for one week.

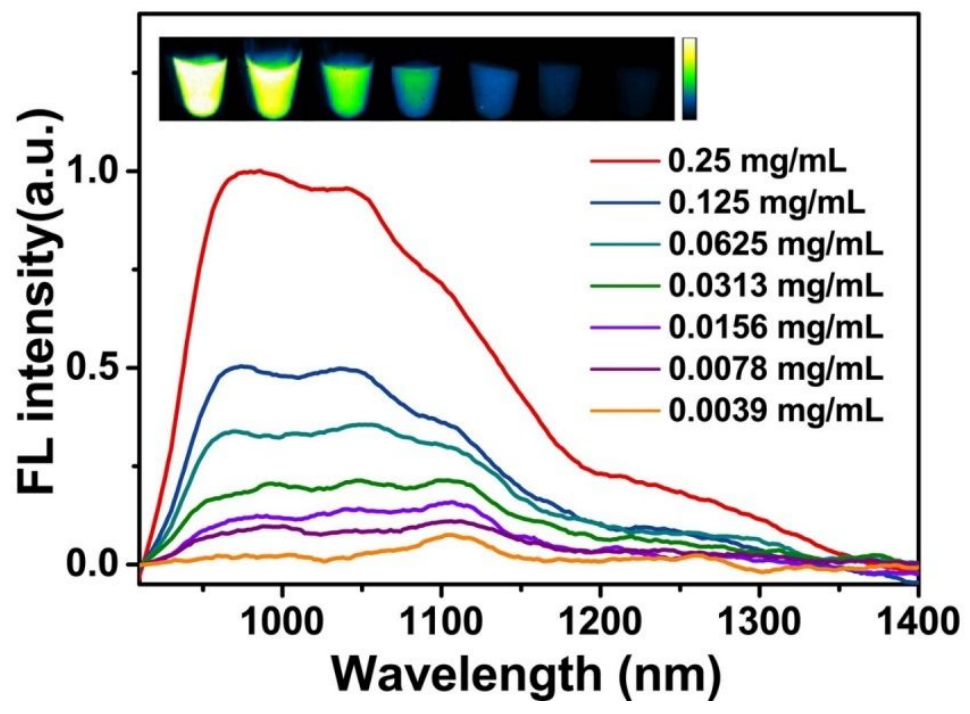

Figure S1. NIR-II fluorescence spectra of different concentration of BPQDs. 


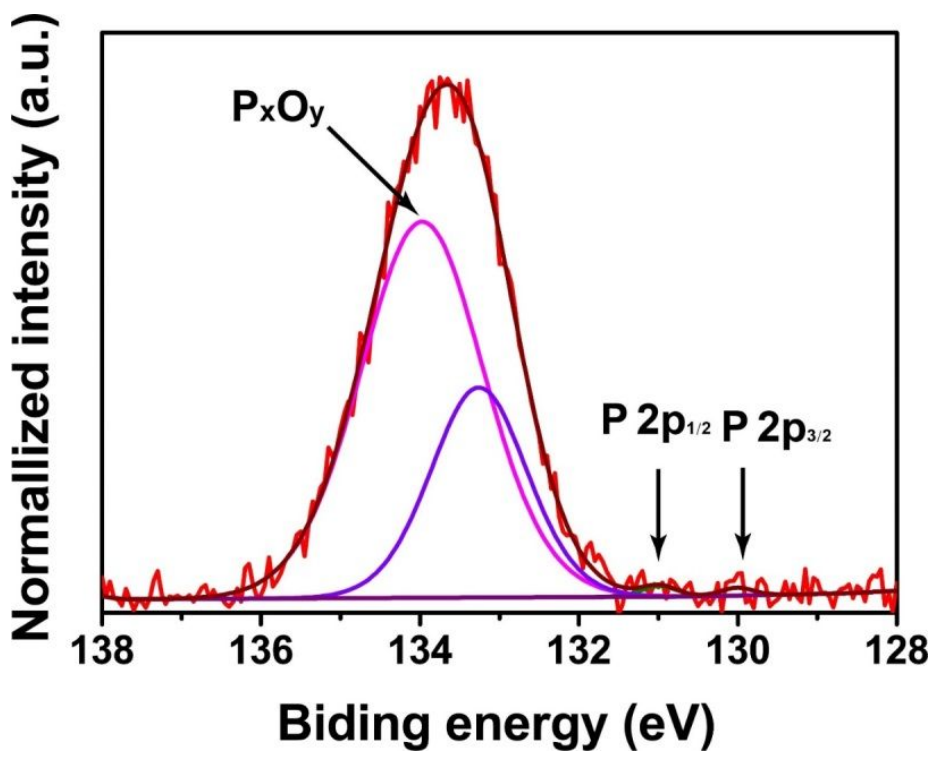

Figure S2. P 2p XPS spectra of the BPQDs at day 8.

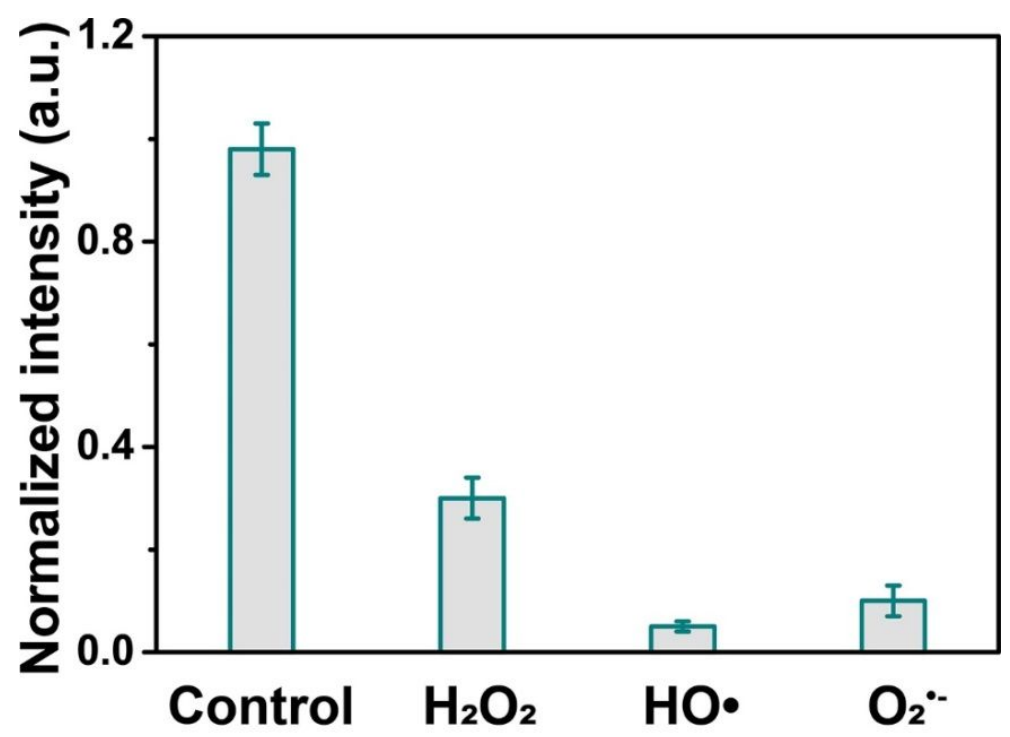

Figure S3. NIR-II fluorescence intensity of BPQDs solutions treated with different kinds of $\operatorname{ROS}\left(\mathrm{H}_{2} \mathrm{O}_{2}, \bullet \mathrm{OH}\right.$, and $\left.\mathrm{O}_{2}{ }^{\bullet-}\right)$. 


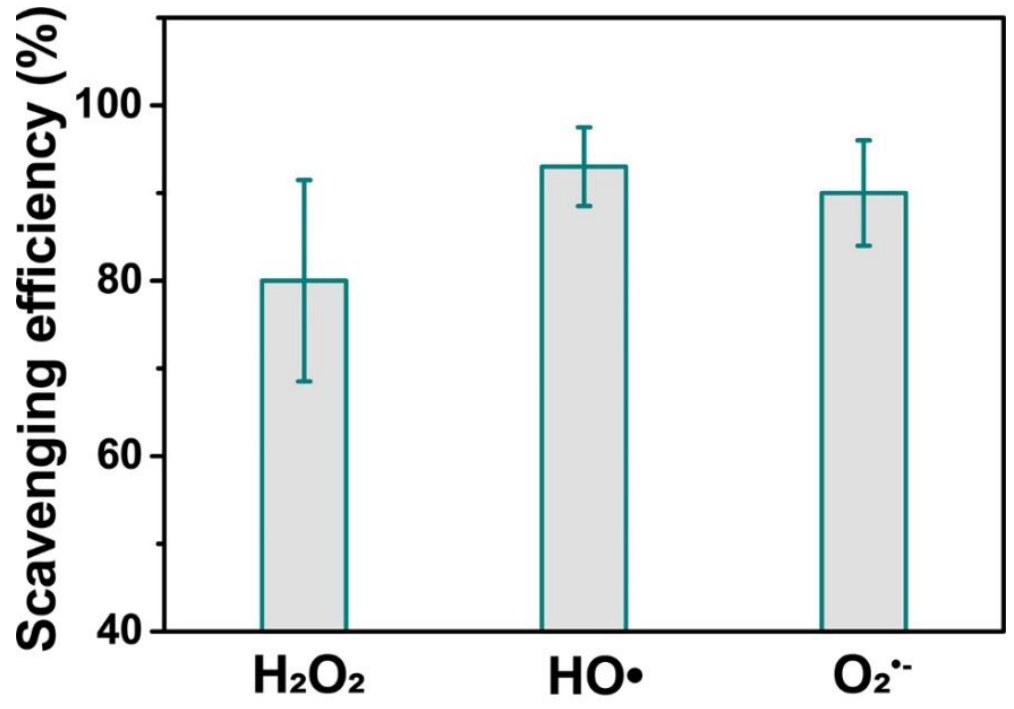

Figure S4. The scavenging efficiency of BPQDs incubated with $\mathrm{H}_{2} \mathrm{O}_{2}, \bullet \mathrm{OH}$, and $\mathrm{O}_{2}{ }^{\bullet}$.

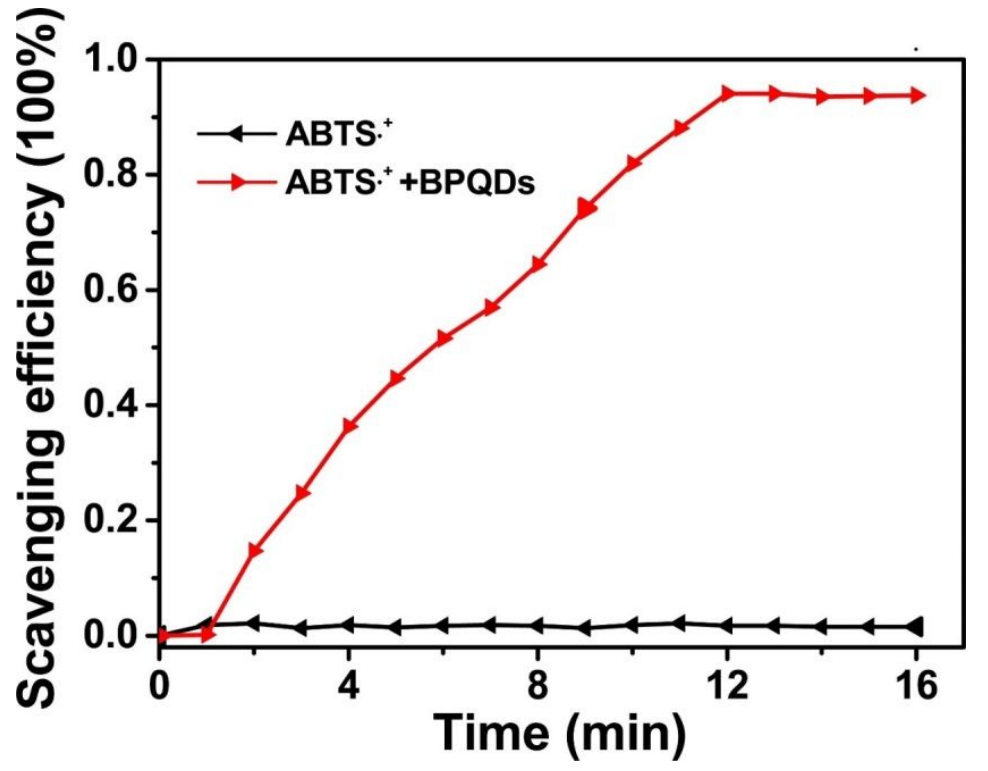

Figure S5. The free radical scavenging efficiency of BPQDs with lapse of time. 

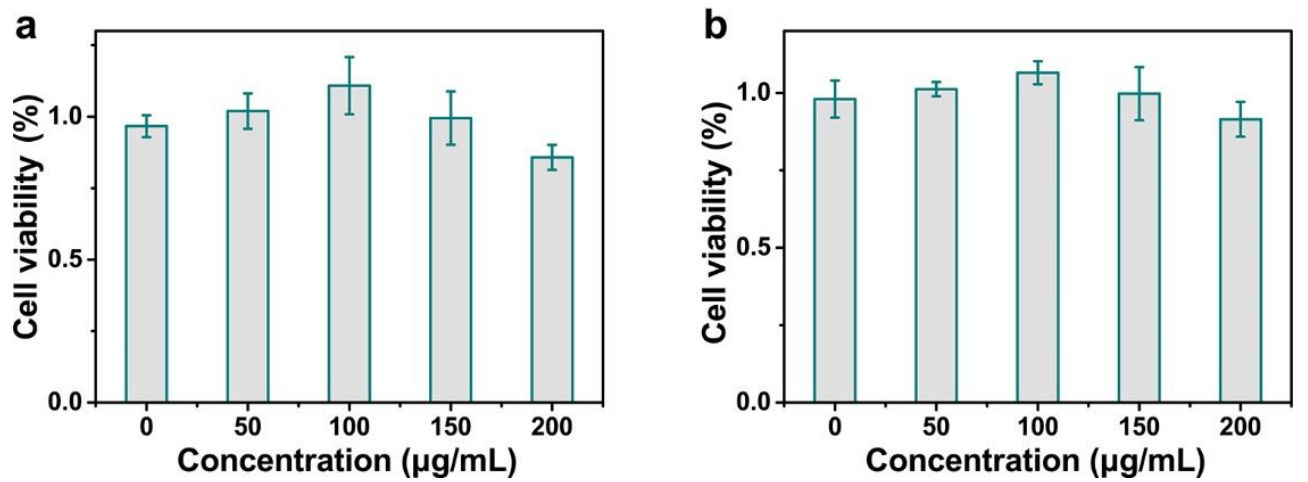

Figure S6. Cell viability of 293T (a) and L02 (b) incubated with BPQDs at different concentrations.

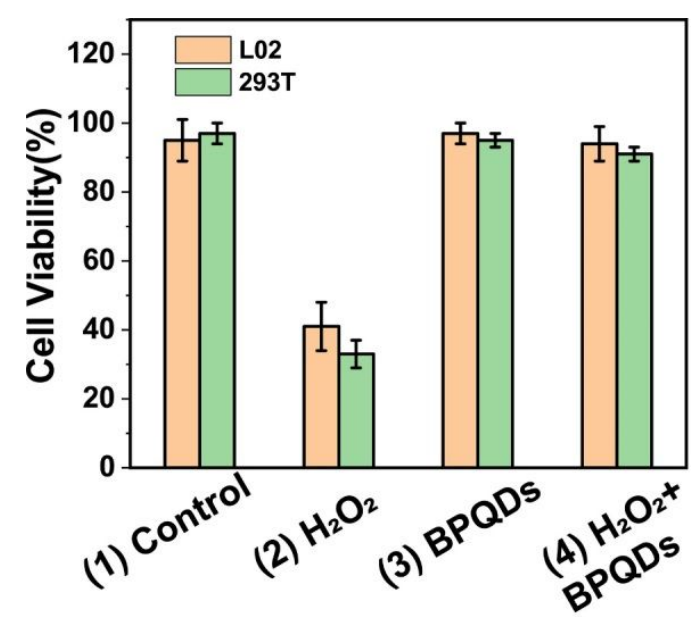

Figure S7. Cell viability of 293T and L02 under different treatment in four groups: (1) control, (2) BPQDs, (3) $\mathrm{H}_{2} \mathrm{O}_{2}$, (4) $\mathrm{H}_{2} \mathrm{O}_{2}+$ BPQDs. 


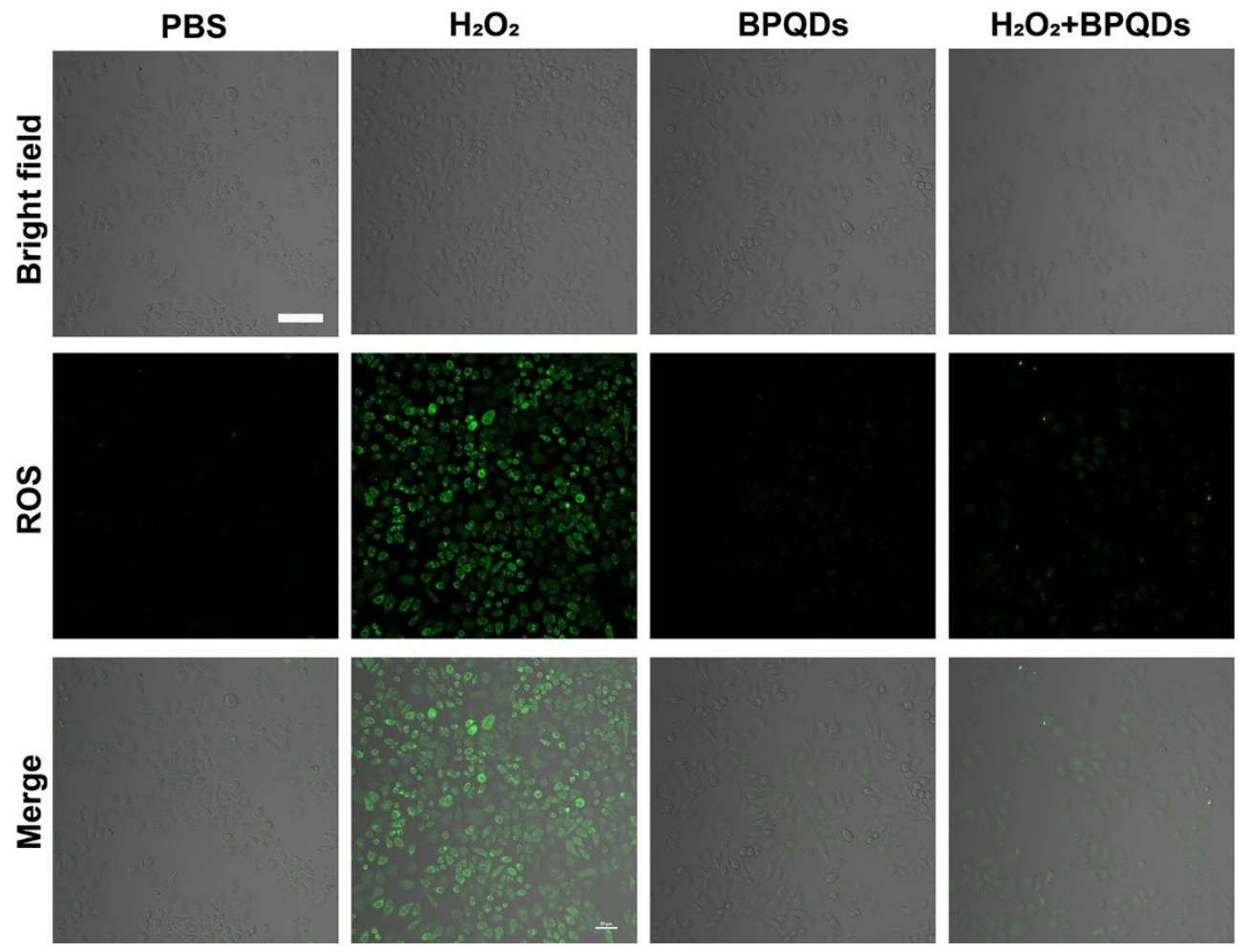

Figure S8. Confocal images of ROS scavenging in cells indicated by DCFH-DA emitting green fluorescence in L02 cells with $1 \mathrm{mM} \mathrm{H}_{2} \mathrm{O}_{2}$. Scale bar: $100 \mu \mathrm{m}$.

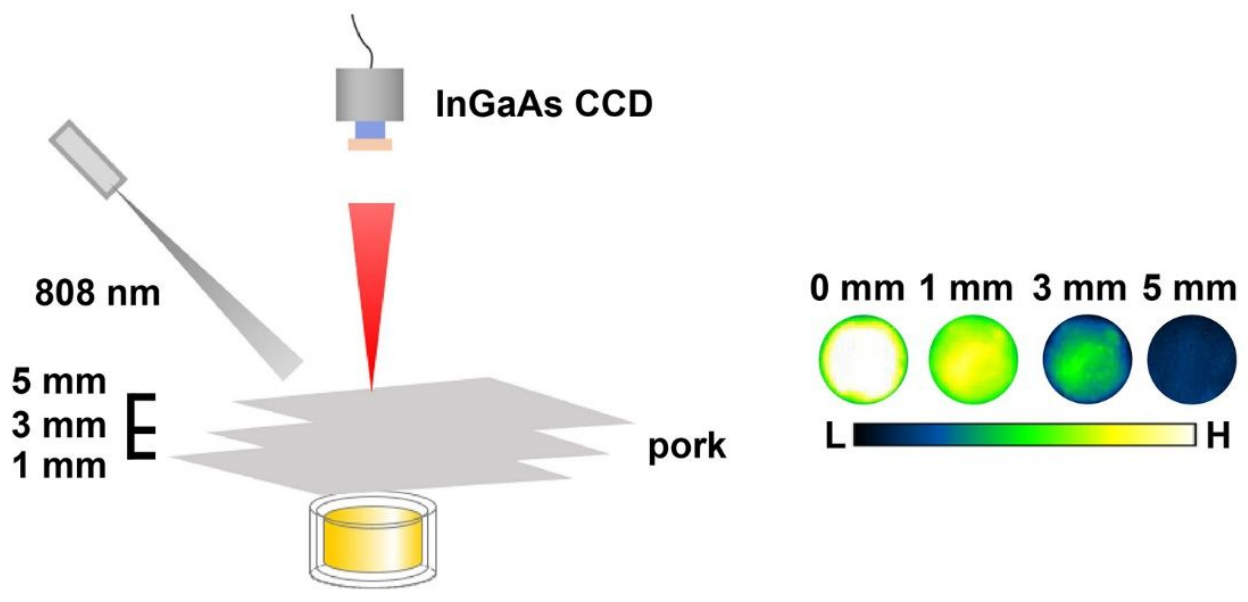

Figure S9. NIR-II fluorescence images at $1050 \mathrm{~nm}$ of 96 -well plates covered by pork with varied thickness of $0,1,3$, and $5 \mathrm{~mm}$. 

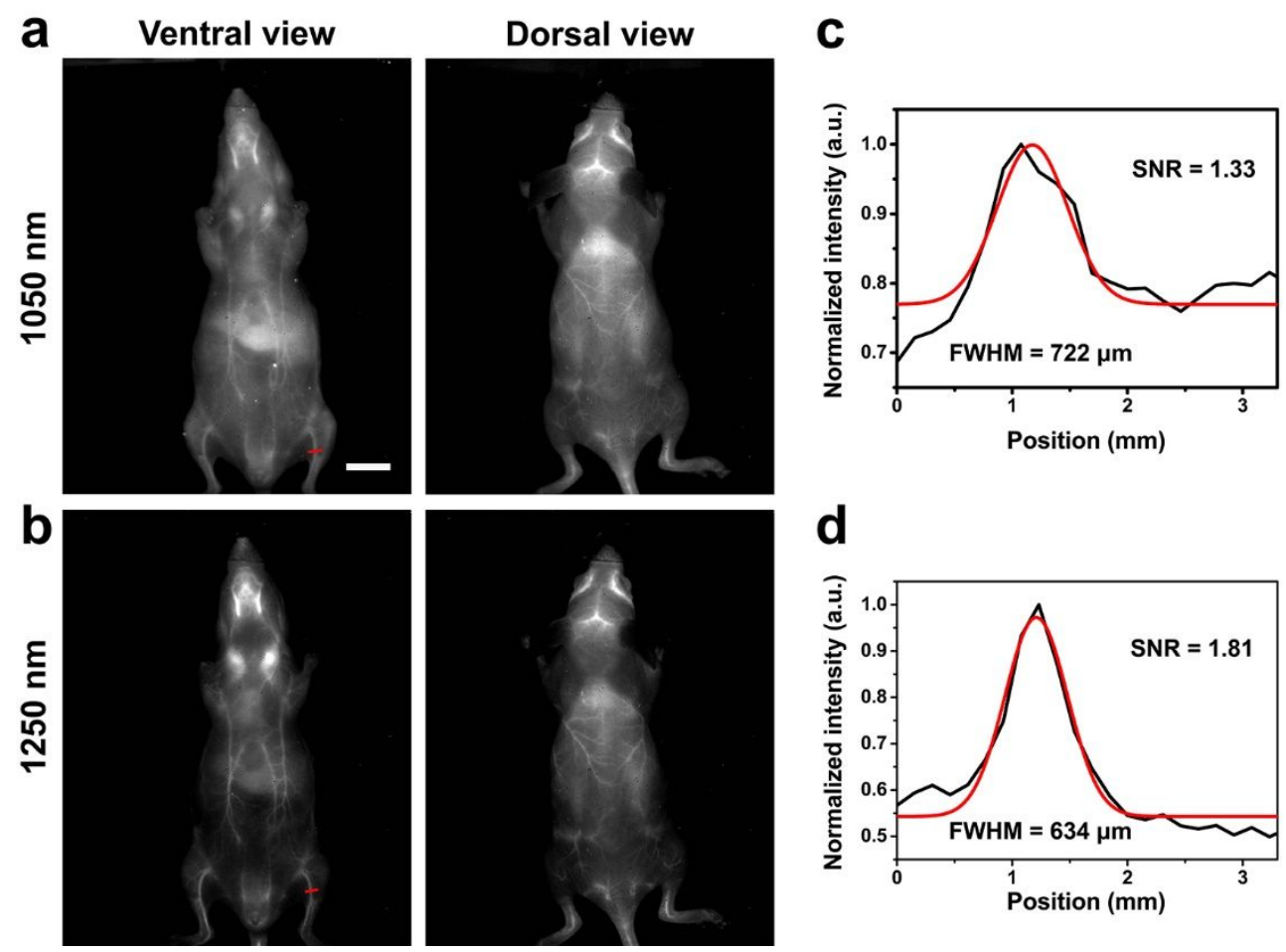

Figure S10. NIR-II fluorescence images at $1050 \mathrm{~nm}$ and $1250 \mathrm{~nm}$ of mice at post-injection $5 \mathrm{~min}$. Scale bar: $1 \mathrm{~cm}$. (a) imaging condition: $1050 \mathrm{~nm}$ band-gap filter, $200 \mathrm{~ms}$ exposure time, and $180 \mathrm{~mW} \mathrm{~cm}^{-2}$. (b) imaging condition: $1250 \mathrm{~nm}$ band-gap filter, $700 \mathrm{~ms}$ exposure time, and $180 \mathrm{~mW} \mathrm{~cm}^{-2}$. (c-d) The normalized fluorescence intensity of blood vessel (red-dashed lines) profiles fitted by Gaussian.
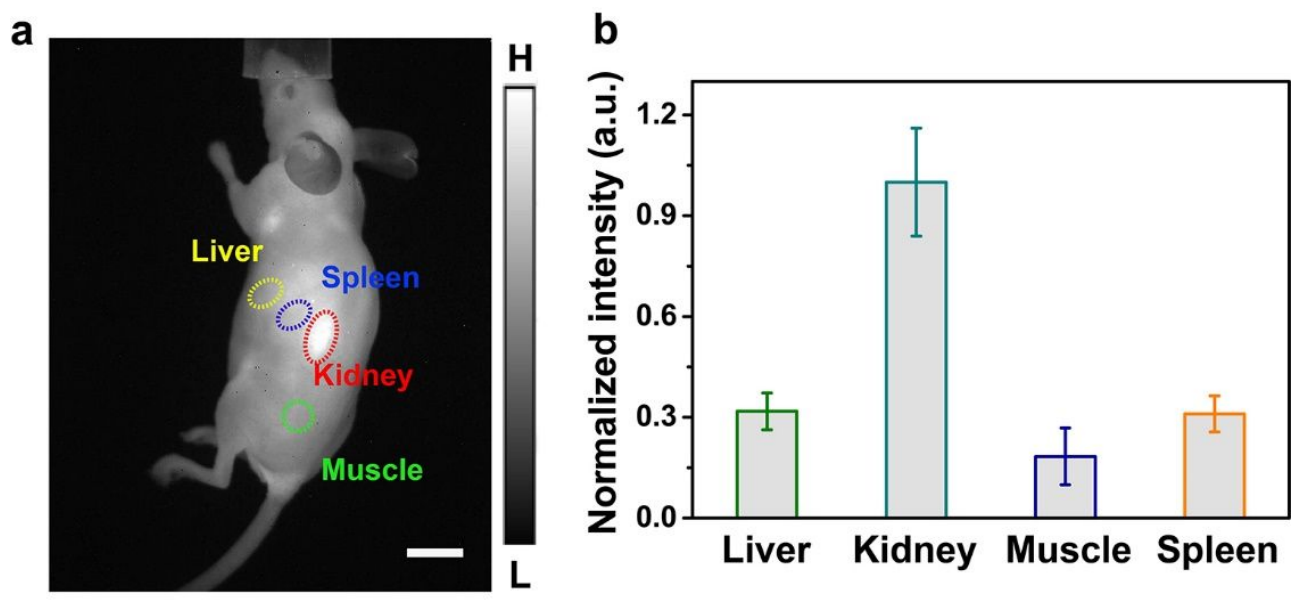

Figure S11. (a) In vivo NIR-II fluorescence images of mice at $6 \mathrm{~h}$ post-injection. (b) corresponding region of interest (ROI) analysis. Scale bar: $1 \mathrm{~cm}$. 


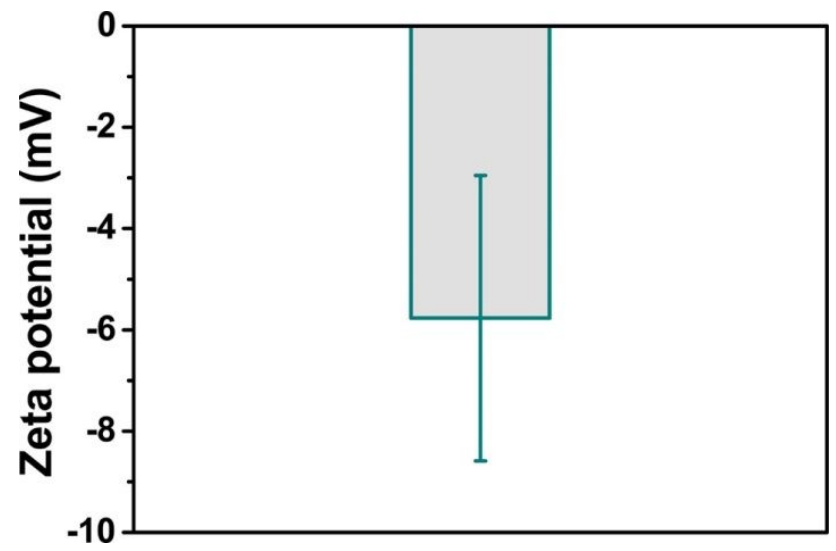

Figure S12. Zeta potential of BPQDs in water at $\mathrm{pH} 7.4\left(25^{\circ} \mathrm{C}\right)$.
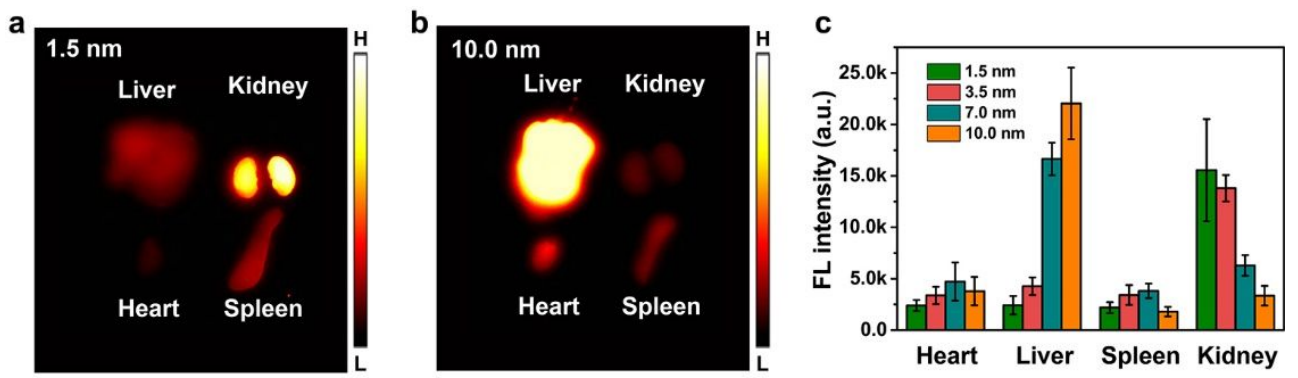

Figure S13. (a) Ex vivo NIR-II FL images of mince treated with BPQDs (the size of BPQDs is $1.5 \mathrm{~nm}$ ). (b) Ex vivo NIR-II FL images of mince treated with BPQDs (the size of BPQDs is $10.0 \mathrm{~nm}$ ). (c) Corresponding region of interest analysis.
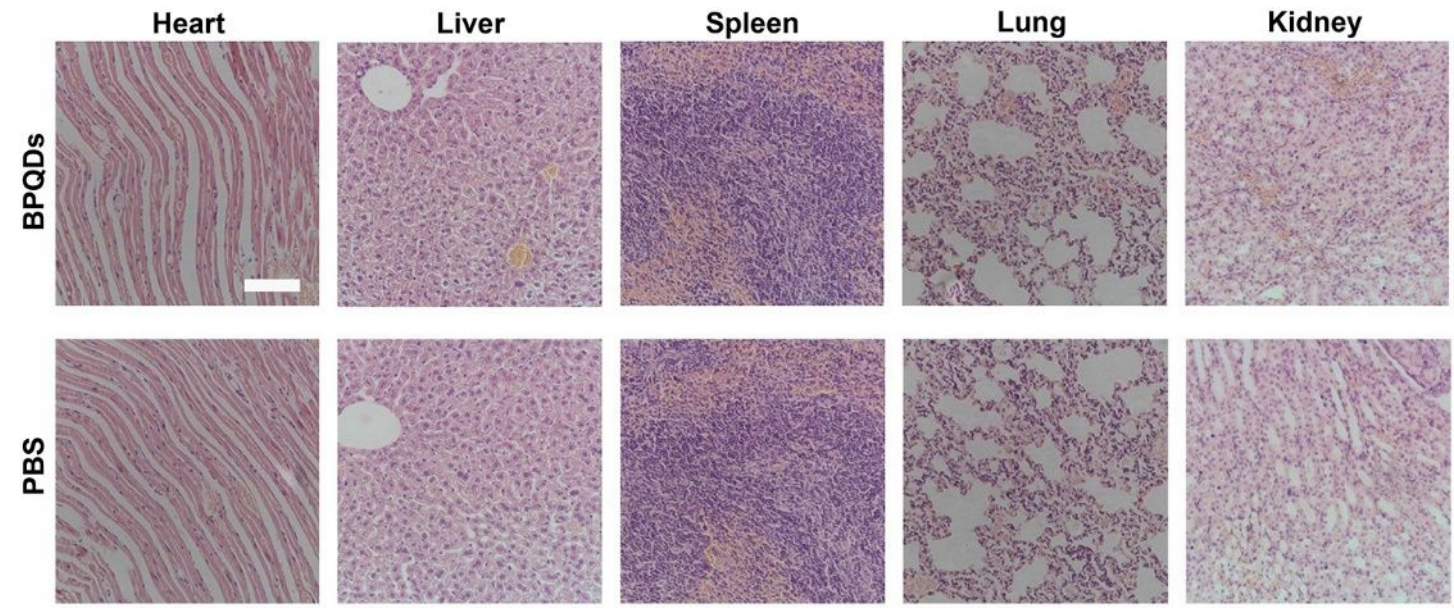

Figure S14. Hematoxylin and eosin staining of main organs from mice treated with BPQDs including heart, kidney, spleen, liver. Scale bar: $200 \mu \mathrm{m}$. 